\title{
Sirt1 plays an important role in mediating greater functionality of human ES/iPS-derived vascular endothelial cells.
}

\section{AUTHOR(S):}

Homma, Koichiro; Sone, Masakatsu; Taura, Daisuke; Yamahara, Kenichi; Suzuki, Yutaka; Takahashi, Kazutoshi; Sonoyama, Takuhiro; ... Itoh, Hiroshi; Yamanaka, Shinya; Nakao, Kazuwa

\section{CITATION:}

Homma, Koichiro ... [et al]. Sirt1 plays an important role in mediating greater functionality of human ES/iPS-derived vascular endothelial cells.. Atherosclerosis 2010, 212(1): 42-47

\section{ISSUE DATE:}

2010-09

URL:

http://hdl.handle.net/2433/130711

\section{RIGHT:}

(c) 2010 Published by Elsevier Ltd; この論文は出版社版でありません。 引用の際には出版社版をご確認ご利用ください。; This is not the published version. Please cite only the published version. 
Sirt1 plays an important role in mediating greater functionality of human ES/iPS derived vascular endothelial cells

Koichiro Homma ${ }^{\mathrm{a}, \mathrm{e}}$, Masakatsu Sone ${ }^{\mathrm{a}^{*}}$, Daisuke Taura ${ }^{\mathrm{a}}$, Kenichi Yamahara $^{\mathrm{a}}$, Yutaka Suzuki ${ }^{\mathrm{b}}$, Kazutoshi Takahashi ${ }^{\mathrm{c}, \mathrm{d}}$, Takuhiro Sonoyama ${ }^{\mathrm{a}}$, Megumi Inuzuka ${ }^{\mathrm{a}}$, Yasutomo Fukunaga ${ }^{\mathrm{a}}$, Naohisa Tamura $^{\mathrm{a}}$, Hiroshi Itoh ${ }^{\mathrm{e}}$, Shinya Yamanaka ${ }^{\mathrm{c}, \mathrm{d}}$, Kazuwa Nakao $^{\mathrm{a}}$

${ }^{a}$ Department of Medicine and Clinical Science, Kyoto University Graduate School of Medicine, Kyoto, Japan

${ }^{\mathrm{b}}$ Stem Cell and Drug Discovery Institute, Kyoto, Japan

${ }^{c}$ Department of Stem Cell Biology, Institute for Frontier Medical Sciences, Kyoto University, Kyoto, Japan

${ }^{\mathrm{d}}$ Center for iPS Cell Research and Application (CiRA), Institute for Integrated Cell-Material Sciences, Kyoto, Japan

${ }^{\mathrm{e}}$ Department of Internal Medicine, Keio University School of Medicine, Tokyo, Japan

\section{*Correspondence to:}

Masakatsu Sone, $\mathrm{MD}, \mathrm{PhD}$

54 Shogoin Kawahara-cho, Sakyo-ku, Kyoto 606-8507, Japan 
Tel: $+81-75-751-3170$

Fax: +81-75-771-9452

E-mail: sonemasa@kuhp.kyoto-u.ac.jp

Total word count of the manuscript: 3938 words 


\begin{abstract}
Objective We previously succeeded in inducing and isolating vascular endothelial cells (ECs) from both human embryonic stem (ES) and induced pluripotent stem (iPS) cells. Here, we compared the functionality of human adult ECs (HAECs), human ES-derived ECs (ESECs) and human iPS-derived ECs (iPSECs).
\end{abstract}

Methods and Results We compared the cell proliferative potential, potential for migration, and tolerance to oxidative stress. ESECs were significantly superior to HAECs in all of these cell functions. The cell functions of iPSECs were comparable to those of ESECSs and also superior to HAECs. We then analyzed the gene expressions of HAECs, ESECs and iPSECs, and observed that the expression level of Sirt1, a nicotinamide adenine dinucleotide (NAD+)-dependent histone deacetylase, is higher in ESECs and iPSECs than in HAECs. The inhibition of Sirt1 with a Sirt1-specific inhibitor and siRNA antagonized these differences between the three types of cells.

Conclusions Sirt1 plays a key role in the high cellular function of ESECs and iPSECs. Although further in vivo investigations are required, this study initially demonstrated the potential of ESECs and iPSECs as the cell source for regenerative medicine, and also showed the potential of ES cells as a useful tool for elucidating the molecular mechanism of cell aging.

Key words

Stem cells, endothelium, Sirt1 


\section{Introduction}

We have succeeded in selectively inducing mouse, monkey and human ES cells to differentiate into ECs and mural cells and in isolating those cells (1-3). In addition, we recently succeeded in inducing human iPS cells to differentiate into ECs and mural cells and in isolating those cells (4). We have also intra-arterially transplanted human ES cell-derived ECs (ESECs) into murine hindlimb ischemia models and found that the transplanted ECs are incorporated into the host vasculature, where they promote the restoration of blood flow. By contrast, almost no transplanted adult aorta-derived ECs were incorporated into the host vasculature, and they did not promote blood flow restoration (3, 5). Other groups comparing the efficiency of engraftment of ESECs and human adult ECs obtained similar results (6-8). Apparently, there are functional differences between ESECs, which are at a relatively early stage of development, and human adult ECs, which have already been subject to aging.

The aims of the present study were to analyze the functional differences between human adult ECs, ESECs and human iPS cells-derived ECs (iPSECs), to identify factors responsible for these functional differences, and to determine at least part of the mechanism of vascular aging. 


\section{Methods}

\section{Cell culture}

Human aortic endothelial cells (HAECs) were purchased from Lonza and maintained in endothelial growth medium (EGM-2, EGM-2 singleQuots, Lonza). Human saphenous vein endothelial cells (HVECs) were purchased from VEC Technologies, Inc., and maintained in EGM-2 (EGM-2 singleQuots). The khES1 human ES cell line and the 201B7 human iPS cell line were used and maintained as described previously (4). Briefly, every 5 to 6 days, undifferentiated cells of both cell lines were detached with dissecting pipettes and transferred to dishes with mitomycin C-treated mouse feeder cells. All endothelial cells used in this study were not passaged more than five times.

\section{Induction of differentiation}

Undifferentiated human ES or iPS cells were harvested and transferred to a collagen I-coated dish after adjusting the colonies to an appropriate size. On the second day of incubation, the culture medium was replaced with human ES/iPS cell maintenance medium without basic FGF, supplemented with N2 supplement (Invitrogen)/B27 supplement (Invitrogen) and BIO (SIGMA). Thereafter, the cells were incubated for another 3 days, at which time the culture medium was replaced with StemPro-34 SFM (Invitrogen) supplemented with VEGF (50 ng/ml; PeproTech EC Ltd). After another 3-5 days of incubation, Flk1/VE-cadherin+/+ cells were sorted using FACSAria flow cytometer and used for the following experiments. Sorted cells were confirmed to remain VE-cadherin positive during the following cell culture and analyses.

\section{Immunohistochemistry}

Cultured cells were stained with an anti-VE-cadherin antibody (BV-9, Abcam) (9), or the indicated monoclonal antibodies as described (4). 


\section{MTT assay}

Cell proliferation was assessed in colorimetric 3,4,5-dimethylthiazol-2-yl-2,5-diphenyl tetrazolium bromide (MTT) assays carried out as described previously (10). Briefly, cells were incubated with MTT (Nakarai Tesque, Kyoto, Japan) solution for $4 \mathrm{~h}$, after which the medium was discarded. The remaining dye was then dissolved in dimethyl sulfoxide, and the absorbance was measured at $570 \mathrm{~nm}$.

\section{In vitro wound healing assay}

Wound healing assays were carried out as we described previously (11). Briely, ECs were grown to overconfluence in six-well plates, after which a wound approximately $2 \mathrm{~mm}$ in width was made with a cell scraper. The wound was then allowed to heal (re-endothelialize) for $24 \mathrm{~h}$ in the same medium. The wounded monolayer was photographed before and after the incubation period, and the area of re-endothelialization was evaluated.

\section{Annexin V assay}

Confluent monolayers (80-90\%) of ECs grown in 6-well plates were treated with or without 300 $\mu \mathrm{mol} / 1 \mathrm{H}_{2} \mathrm{O}_{2}$. After $8 \mathrm{~h}$ of exposure, annexin V-FITC in combination with Via-Probe was used to quantitatively determine the percentage of cells undergoing apoptosis, as described previously (12). Briefly, after treating the cells with the indicated reagents, the monolayer was detached by a brief incubation with Trypsin-EDTA solution. Aliquots of cells $\left(10^{5}\right)$ were then resuspended in $1 \mathrm{x}$ binding buffer (BD Pharmingen, San Diego, CA) and incubated with annexin V-FITC for 15 min at room temperature in the dark, stained with Via-Probe, and analyzed within $1 \mathrm{~h}$ in a FACSAria flow cytometer. FACS Diva software (Becton Dickinson) was used to analyze the data. Early apoptotic cells were stained with annexin V only, while late apoptotic or necrotic cells were stained with both annexin V and Via-Probe. 


\section{Quantitative real-time PCR}

Total RNA was isolated using an RNeasy ${ }^{\mathbb{B}}$ Mini Kit and treated with an RNase-Free DNase Set (QIAGEN, Germany) to remove any contaminating genomic DNA. Quantitative real-time PCR was then performed using Premix ExTaqTM(Takara Bio Inc., Shiga, Japan). The PCR primers used were as follows: for Sirt1, GCCTCACATGCAAGCTCTAGTGAC(forward) and TTCGAGGATCTGTGCCAATCATAA(reverse); for Delta-like 4 (DLL4), GTGGACTGTGGCCTGGACAA(forward) and AGCATATCGCTGATATCCGACACACTC(reverse); for CXCR4, GCCAACGTCAGTCAGTGAGGCAGA(forward) and GCCAACCATGATGTGCTGAAAC(reverse) and for $\beta$-actin, CATCCGTAAAGACCTCTATGCCAC(forward) and ATGGAGCCACCATCCACA(reverse). All primers were pruduced by Takara Bio. Levels of Sirt1, DLL4 and CXCR4 mRNA are presented after normalization to the level of $\beta$-actin mRNA.

\section{Western blot analysis}

Western blotting was carried out using a standard protocol described previously (13). Anti-Sirt1 antibody was purchased from Santa Cruz Biotechnology, Inc.

\section{SiRNA transfection}

Small interference RNA (siRNA) against Sirt1 and negative control were provided by Qiagen. The target sequence for the Sirt1 siRNA was 5'CAA GCG ATG TTT GAT ATT GAA3'. ECs were trypsinized, washed with Hank's Balanced Salt Solution, resuspended $\left(5 \times 10^{5}\right.$ cells $)$ in human umbilical vein endothelial cell solution (Amaxa Biosystems) containing $3 \mu \mathrm{g}$ of siRNA duplex, and then transfected using a Nucleofector (Amaxa Biosystems) following the manufacturer's instructions. After transfection, the cells were immediately plated in dishes.

\section{Endothelial tube formation assay}


Endothelial tube formation was assayed as described previously (14). ECs (20,000 cells/well) were seeded into Matrigel-coated 24-well plates. The cells were then incubated for $12 \mathrm{~h}$ at $37^{\circ} \mathrm{C}$, after which the formed tubes were digitally imaged and analyzed using MetaMorph soft ware (Universal Imaging Corp.).

\section{Statistical analysis.}

Results are presented as means \pm SEM. Differences between groups were analyzed using ANOVA followed by Fisher`s analysis for comparisons between two means. Values of $\mathrm{P}<0.05$ were considered significant.

\section{Results}

\section{Morphological comparison of HAECs, ESECs and iPSECs}

HAECs, ESECs and iPSECs were morphologically similar in that they all exhibited a cobblestone-like appearance on culture dishes, were positive for eNOS, and showed a marginal staining pattern when stained for CD31 and VE-cadherin (Figure 1). All of these features are characteristic of vascular endothelial cells. Furthermore, HVECs, an example of venous endothelial cells, were analyzed together with HAECs, ESECs and iPSECs by real-time PCR for the expression of arterial endothelial marker genes, DLL4 and CXCR4. The expression levels of DLL4 and CXCR4 in both ESECs and iPSECs were comparable with those in HAECs and higher than those in HVECs (supplementary Figure I), suggesting that both ESECs and iPSECs are near artery-lineage.

\section{Comparison of the potentials for proliferation and migration and tolerance for oxidative stress of HAECs, ESECs and iPSECs}

MTT assays carried out to evaluate their proliferative potential revealed ESECs and iPSECs to have a significantly greater potential for proliferation than HAECs, but there was no significant 
difference between ESECs and iPSECs (Figure 2A). Similarly, an in vitro wound healing assay revealed that ESECs and iPSECs to have a significantly higher potential for migration after EC loss than HAECs, and again there was no significant difference between ESECs and iPSECs (Figure 2B). Following induction of oxidative stress by exposure to $\mathrm{H}_{2} \mathrm{O}_{2}$, moreover, there was a significantly lower percentage of annexin V-positive apoptotic cells among ESECs and iPSECs than among HAECs, with no significant difference between ESECs and iPSECs (Figure 2C).

\section{Differences in the expression of Sirt1 in HAECs, ESECs and iPSECs}

To identify factors responsible for the functional differences between HAECs, ESECs and iPSECs, we next carried out a gene expression analysis using gene chip technology (supplementary Method, supplementary Figure II). We found that the expression level of Sirt1, an aging-related gene that encodes a NAD-dependent histone deacetylase, was higher in both ESECs and iPSECs than in HAECs. We then confirmed that finding by using real-time PCR to quantitatively compare the levels of Sirt1 mRNA expression in the three cell types. Sirt1 mRNA was expressed at significantly higher levels in both ESECs and iPSECs than in HAECs (Figure 2D), but there was no significant difference in expression between ESECs and iPSECs. Subsequent Western blot analysis of Sirt1 protein expression yielded analogous results (Figure 2E).

\section{Contribution of Sirt1 to the cellular functionality of HAECs, ESECs and iPSECs}

In view of the possibility that differences in Sirt1 expression contributes to the observed differences in the cellular functionality of HAECs, ESECs and iPSECs, we examined the effects of knocking down Sirt1 expression using siRNA or inhibiting Sirt1 protein using sirtinol (Calbiochem, San Diego, CA), a specific Sirt1 antagonist $(15,16)$. Using real-time PCR, we found that the targeted siRNA reduced the level of Sirt1 mRNA by about $70 \%$, as compared to the nontargeted siRNA (data not shown). Although under control conditions ESECs and iPSECs showed a significantly greater proliferative potential than HAECs, knocking down Sirt1 expression diminished 
proliferation of all three cell types and abolished all differences in proliferative potential (Figure 3A). Likewise, the difference in the cells' potential for migration also disappeared when Sirt1 was knocked down (Figure 3B). Similar results were obtained when $50 \mu \mathrm{M}$ sirtinol was applied to the cells (Figure 3A upper, 3B upper). The vehicle control was $0.025 \%$ DMSO. Because sirtinol was dissolved in DMSO. On the other hand, differences in tolerance for oxidative stress became nonsignificant, but the trend remained upon Sirt1 knock down (Figure 3C).

Changes in Sirt1 expression level during differentiation towards endothelial cells, and effects of Sirt1 on endothelial cell differentiation

To investigate the involvement of Sirt1 in the differentiation process towards endothelial cells, Sirt1 gene expression level was determined by real-time PCR before and after VEGF stimulation. The level of Sirt1 expression in differentiated mesoblastic cells before VEGF addition was not significantly different from that in the VEGF-stimulated cells immediately before cell sorting (supplementary Figure III A). Furthermore, inhibition of Sirt1 by the addition of $25 \mathrm{mM}$ or $50 \mathrm{mM}$ sirtinol during the induction period did not alter endothelial cell differentiation efficiency (supplementary Figure III B).

\section{Contribution of Sirt1 to the angiogenic activities of HAECs, ESECs and iPSECs}

Finally, we used Matrigel assays to determine whether the differences in the functionality of the three cell types are reflected in their angiogenic activities. Consistent with the results summarized above, ESECs and iPSECs showed greater tube formation than HAECs, but that difference was abolished by knocking down Sirt1 expression (Figure 4, supplementary video 1, 2). Similar results were obtained when $50 \mu \mathrm{M}$ sirtinol was applied to the cells (data not shown). 


\section{Discussion}

The results of the present study demonstrate that human ESECs have a greater potential for proliferation and migration and a greater tolerance for oxidative stress than HAECs. They also demonstrate that the functionality of iPSECs is similar to that of ESECs.

Gene chip analysis and quantitative comparison using real-time PCR revealed that levels of Sirt1 expression differed between ESECs and HAECs. Sirt1, a mammalian homologue of Sir2, has recently been drawing attention due to its relationship to endothelial function (14-17) and eNOS expression in ECs (18). It has also been reported that Sirt1 is important for the normal function of not only mature endothelial cells but also endothelial progenitor cells (19). All of these previous studies demonstrated a protective role of Sirt1 in endothelial regeneration. In the present study, we found that when Sirt1 activity was knocked down by siRNA or inhibited by a specific Sirt1 antagonist, the aforementioned differences in the cellular functionality of ESECs and HAECs were abolished, which suggests that differences in Sirt1 activity contribute significantly to the observed differences in the cellular functionality of the two cell types. Moreover, this implies that changes in Sirt1 expression play a key role in mediating the effects of aging on human EC function, and that intervention to regulate Sirt1 expression may represent a useful approach to slowing the aging of ECs and improving their functionality.

Cell proliferation and migration are important steps in the process of angiogenesis. Sirt1 was previously shown to promote cell proliferation by suppressing p53 (20) and to promote cell migration through deacetylation of cortactin (21). The differences in their potentials for proliferation and migration are also likely reflected in the results of the in vitro tube formation assays performed to assess the cells' angiogenic potential. Furthermore, the Matrigel assay showed strongly impaired tube formation due to the suppression of Sirt1 in all three cell types. This suggests that Sirt1 may play an essential role in endothelial sprouting.

The post-transplant engraftment rate is another important indicator of a cell's utility for transplantation therapy. Several studies have shown that the engraftment rate is closely related to the 
incidence of apoptosis induced by inflammatory cytokines produced in inflamed or injured tissues (22). Thus the observed anti-apoptotic effect in ESECs and iPSECs may be indicative of their potential for use in cell transplantation therapy. Nonsignificant but residual differences in tolerance for oxidative stress remained after suppressing Sirt1, suggesting the possible involvement of other factors. The protective effect of Sirt1 against oxidative stress has also been observed by several other studies (15, 16, 23). Balestrieri et al. reported that metabolic intervention enhances therapeutic effects achieved by the administration of bone marrow cells or hemangioblasts alone in a mouse model of hindlimb ischemia (24). In vivo experiments investigating the actual efficacy of a therapy using Sirt1 as a target are anticipated in the future.

The present and previous studies clearly demonstrated that Sirt1 plays an important role in endothelial cell function; however, whether it is involved in endothelial cell differentiation remains unclear. In this study, we showed that Sirt1 expression level was unchanged during induction, and that Sirt1 inhibition during induction did not alter the endothelial cell differentiation efficiency. Therefore, at least in the in vitro induction protocol used in the present study, Sirt1 appeared to have no effect on endothelial cell differentiation.

We found that iPSECs are very similar to ESECs, as judged from their expression of aging-related genes and their cellular functionality. iPS cells originate from somatic cells and are dedifferentiated through reprogramming. This is noteworthy, as it suggests that cells differentiated from iPS cells (e.g., iPSECs) are relieved of the aging they experienced as somatic cells so that they are functionally as young as cells differentiated from ES cells. This highlights the importance of reprogramming technology and the elucidation of the underlying mechanisms. In addition, in contrast to human ES cells, iPS cells can be established from every human being irrespective of their genetic backgrounds. The establishment of iPS cell lines from patients with inherited diseases presenting vascular abnormality should enable clarification of their pathogenesis. The establishment of iPS cell lines from patients with various genetic backgrounds should make it possible to dissect out cellular mechanisms in human vascular development, aging and diseased states such as 
arteriosclerosis.

In conclusion, we have shown that ESECs and iPSECs are younger and more viable than HAECs and are thus potentially useful cellular materials for vascular regeneration. We also showed that the functional differences between ESECs/iPSECs and HAECs is mediated by Sirt1 expression, suggesting Sirt1 plays a pivotal role in aging-associated functional impairment of human ECs.

\section{Funding}

This work was supported by the project for realization of regenerative medicine of the Ministry of Education, Culture, Sports, Science and Technology, Japan.

\section{Acknowledgments}

The anti-human Flk1 antibody (KM1668) was a generous gift from Kyowa Hakko Co Ltd. Human ES cell line KhES-1 was obtained from the Institute for Frontier Medical Science, Kyoto University (Kyoto, Japan). This work was supported by the Project for Realization of Regenerative Medicine of the Ministry of Education, Culture, Sports, Science and Technology (MEXT), Japan. This work was also supported by Grants-in-Aid for Scientific Research from the Ministry of Health, Labor and Welfare, MEXT, the Takeda Science Foundation, the Japan Cardiovascular Research Foundation, and the Smoking Research Foundation. We thank Yoshie Fukuchi and Sonoko Takesue for technical assistance. 


\section{References}

1. Yamashita J, Itoh H, Hirashima M, Ogawa M, Nishikawa S, Yurugi T, Naito M, Nakao K and Nishikawa S. Flk1-positive cells derived from embryonic stem cells serve as vascular progenitors. Nature. 2000;408: 92-96.

2. Sone M, Itoh H, Yamashita J, Yurugi-Kobayashi T, Suzuki Y, Kondo Y, Nonoguchi A, Sawada N, Yamahara K, Miyashita K, Park K, Shibuya M, Nito S, Nishikawa S and Nakao K. Different differentiation kinetics of vascular progenitor cells in primate and mouse embryonic stem cells. Circulation. 2003;107:2085-2088.

3. Sone M, Itoh H, Kenichi Yamahara, Yamashita J, Yurugi-K T, Nonoguchi A, Suzuki Y, Chao TH, Sawada N, Fukunaga Y, Miyashita K, Park K, Oyamada N, Sawada N, Taura D, Tamura N, Kondo Y, Nito S, Suemori H, Nakatsuji N, Nishikawa N and Nakao K. Pathway for differentiation of human embryonic stem cells to vascular cell components and their potential for vascular regeneration. Arterioscler Thromb Vasc Biol. 2007;27:2127-2134.

4. Taura D, Sone M, Homma K, Oyamada N, Takahashi K, Tamura N, Yamanaka S and Nakao N. Induction and Isolation of Vascular Cells from Human Induced Pluripotent Stem Cells. Arterioscler Thromb Vasc Biol. 2009 ;29:1100-1103.

5. Yamahara K, Sone M, Itoh H, Yamashita J, Yurugi-Kobayashi T, Homma K, Chao TH, Miyashita K, Park K, Oyamada N, Sawada N, Taura D, Fukunaga Y, Tamura N and Nakao K. Augmentation of Neovascularizaition in hindlimb ischemia by combined transplantation of human embryonic stem cells-derived endothelial and mural cells. PLOS one. 2008;3:e1666.

6. Seung-Woo Cho, Sung-Hwan Moon, Soo-Hong Lee, Sun-Woong Kang, Jumi Kim, Jae Min Lim, Hyo-Soo Kim, Byung-Soo Kim and Hyung- Min Chung. Improvement of Postnatal Neovascularization by Human Embryonic Stem Cell-Derived Endothelial-Like Cell Transplantation in a Mouse Model of Hindlimb Ischemia. Circulation. 2007;116:2409-2419

7. Lu SJ, Feng Q, Caballero S, Chen Y, Moore MA, Grant MB and Lanza R. Generation of functional hemangioblasts from human embryonic stem cells. Nat Methods. 2007;4:501-509. 
8. Yang C, Zhang ZH, Li ZJ, Yang RC, Qian GQ and Han ZC. Enhancement of neovascularization with cord blood CD133Rcell-derived endothelial progenitor cell transplantation. Thromb Haemost. 2004;91:1202-1212.

9. Ma C, Rong Y, Radiloff DR, Datto MB, Centeno B, Bao S, Cheng AW, Lin F, Jiang S, Yeatman TJ, Wang XF. Extracellular matrix protein betaig-h3/TGFBI promotes metastasis of colon cancer by enhancing cell extravasation. Genes Dev. 2008;22:308-321

10. Mosmann T. Rapid colorimetric assay for cellular growth and survival: application to proliferation and cytotoxicity assays. J Immunol Methods. 1983;65:55-63.

11. Miyashita K, Itoh H, Sawada N, Fukunaga Y, Sone M, Yamahara K, Yurugi-Kobayashi T, Park K and Nakao K. Adrenomedullin provokes endothelial Akt activation and promotes vascular regeneration both in vitro and in vivo. FEBS Lett. 2003;544:86-92.

12. Schaefer MB, Wenzel A, Fischer T, Braun-Dullaeus RC, Renner F, Dietrich H, Schaefer CA, Seeger W and Mayera K. Fatty acids differentially influence phosphatidylinositol 3-kinase signal transduction in endothelial cells: Impact on adhesion and apoptosis. Atherosclerosis. 2008;197:630-637.

13. Sawada N, Itoh H, Yamashita J, Doi K, Inoue M, Masatsugu K, Fukunaga Y, Sakaguchi S, Sone M, Yamahara K, Yurugi T and Nakao K. cGMP-dependent protein kinase phosphorylates and inactivates RhoA.Biochem. Biophys. Res. Commun. 2001;280:798-805.

14. Potente M, Ghaeni L, Baldessari D, Mostoslavsky R, Rossig L, Dequiedt F, Haendeler DJ, Mione M, Dejana E, Alt FW, Zeiher AM and Dimmeler S. SIRT1 controls endothelial angiogenic functions during vascular growth. Genes \& Dev. 2007;21:2644-2658.

15. Ota H, Eto M, Kano MR, Ogawa S, Iijima K, Akishita M and Ouchi Y. Cilostazol inhibits oxidative stress-induced premature senescence via upregulation of Sirt1 in human endothelial cells. Arterioscler Thromb Vasc Biol. 2008;28:1634-1639.

16. Ota H, Akishita M, Eto M, Iijima K, Kaneki M and Ouchi Y. Sirt1 modulates premature senescence-like phenotype in human endothelial cells. J Mol Cell Cardiol. 2007;43:571-579. 
17. Gracia-Sancho J, Villarreal G Jr, Zhang Y, García-Cardeña G. Activation of SIRT1 by resveratrol induces KLF2 expression conferring an endothelial vasoprotective phenotype. Cardiovasc Res. 2009;Nov 12

18. Potente M and Dimmeler S. NO Targets SIRT1: A Novel Signaling Network in Endothelial Senescence. Arterioscler. Thromb. Vasc. Biol. 2008;28:1577-1579.

19. Balestrieri ML, Rienzo M, Felice F, Rossiello R, Grimaldi V, Milone L, Casamassimi A, Servillo L, Farzati B, Giovane A, Napoli C. High glucose downregulates endothelial progenitor cell number via SIRT1. Biochim Biophys Acta. 2008;1784:936-45.

20. Chua KF, Mostoslavsky R, Lombard DB, Pang WW, Saito S, Franco S, Kaushal D, Cheng HL, Fischer MR, Stokes N, Murphy MM, Appella E and Alt FW. Mammalian SIRT1 limits replicative life span in response to chronic genotoxic stress. Cell Metab. 2005;2:67-76.

21. Zhang Y, Zhang M, Dong H, Yong S, Li X, Olashaw N, Kruk PA, Cheng JQ, Bai W, Chen J, Nicosia SV and Zhang X. Deacetylation of cortactin by SIRT1 promotes cell migration. Oncogene. 2009;28:445-460.

22. Robey TE, Saiget MK, Reinecke H and Murry CE. Systems approaches to preventing transplanted cell death in cardiac repair. J Mol Cell Cardiol. 2008;45: 567-581.

23. Csiszar A, Labinskyy N, Podlutsky A, Kaminski PM, Wolin MS, Zhang C, Mukhopadhyay P, Pacher P, Hu F, de Cabo R, Ballabh P and Ungvari Z. Vasoprotective effects of resveratrol and SIRT1: attenuation of cigarette smoke-induced oxidative stress and proinflammatory phenotypic alterations. Am J Physiol Heart Circ Physiol. 2008;294:H2721-H2735.

24. Balestrieri ML, Lu SJ, de Nigris F, Giovane A, Williams-Ignarro S, D'Armiento FP, Feng Q, Fiorito C, Testa G, Pastore L, Cacciatore F, Mancini FP, Servillo L, De Rosa G, Pagliarulo C, Rienzo M, Minucci PB, Farzati B, Salvatore F, Rengo F, Ignarro LJ, Giordano A, Baker A, Lanza R, Napoli C. Therapeutic angiogenesis in diabetic apolipoprotein E-deficient mice using bone marrow cells, functional hemangioblasts and metabolic intervention. Atherosclerosis. 2009; Oct 29. 
Figure legends

Figure 1. Phenotypes of HAECs, ESECs and iPSECs.

Typical morphology of an EC monolayer and immunofluorescence staining of the monolayer for CD31, eNOS and VE-cadherin; cell nuclei are stained with DAPI. Scale bar, $100 \mu \mathrm{m}$.

Figure 2. Comparison of the potential for proliferation and migration, tolerance for oxidative stress, and Sirt1 expression in HAECs, ESECs and iPSECs.
(A) MTT assay $(\mathrm{n}=8 ; * \mathrm{P}<0.05)$
(B) Wound healing assay $(\mathrm{n}=4 ; * \mathrm{P}<0.05)$
(C) Annexin V assay $(\mathrm{n}=4-6 ; * \mathrm{P}<0.05)$
(D) Quantitative real-time PCR $(\mathrm{n}=8 ; * \mathrm{P}<0.05)$
(E) Western blot analysis $(\mathrm{n}=4 ; * \mathrm{P}<0.05)$

Figure 3. Effects of Sirt1 siRNA or a Sirt1 inhibitor on the potential for proliferation and migration, and tolerance for oxidative stress in HAECs, ESECs and iPSECs.
(A)MTT assay $(\mathrm{n}=6-8 ; * \mathrm{P}<0.05)$
(B)Wound healing assay $(\mathrm{n}=4 ; * \mathrm{P}<0.05)$
(C)Annexin V assay $(\mathrm{n}=4-6 ; * \mathrm{P}<0.05)$

Figure 4. Tube formation by HAECs, ESECs and iPSECs after $12 \mathrm{~h}$ of culture on Matrigel.
(A) Representative photomicrographs; scale bar, $100 \mu \mathrm{m}$.
(B) Quantitative analysis of tube formation $(\mathrm{n}=4$; $* \mathrm{P}<0.05)$ 

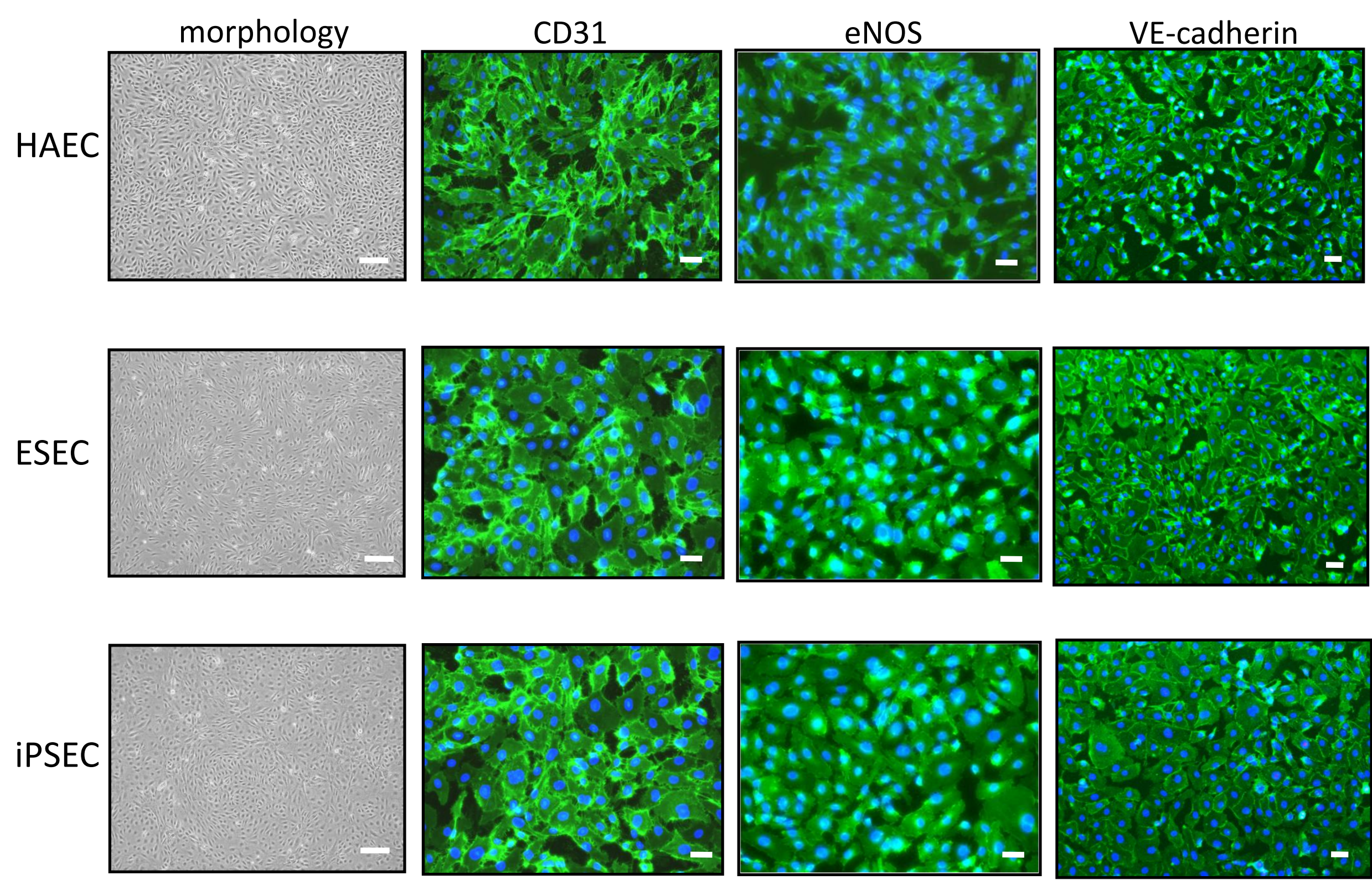
용. 京都大学 3

A
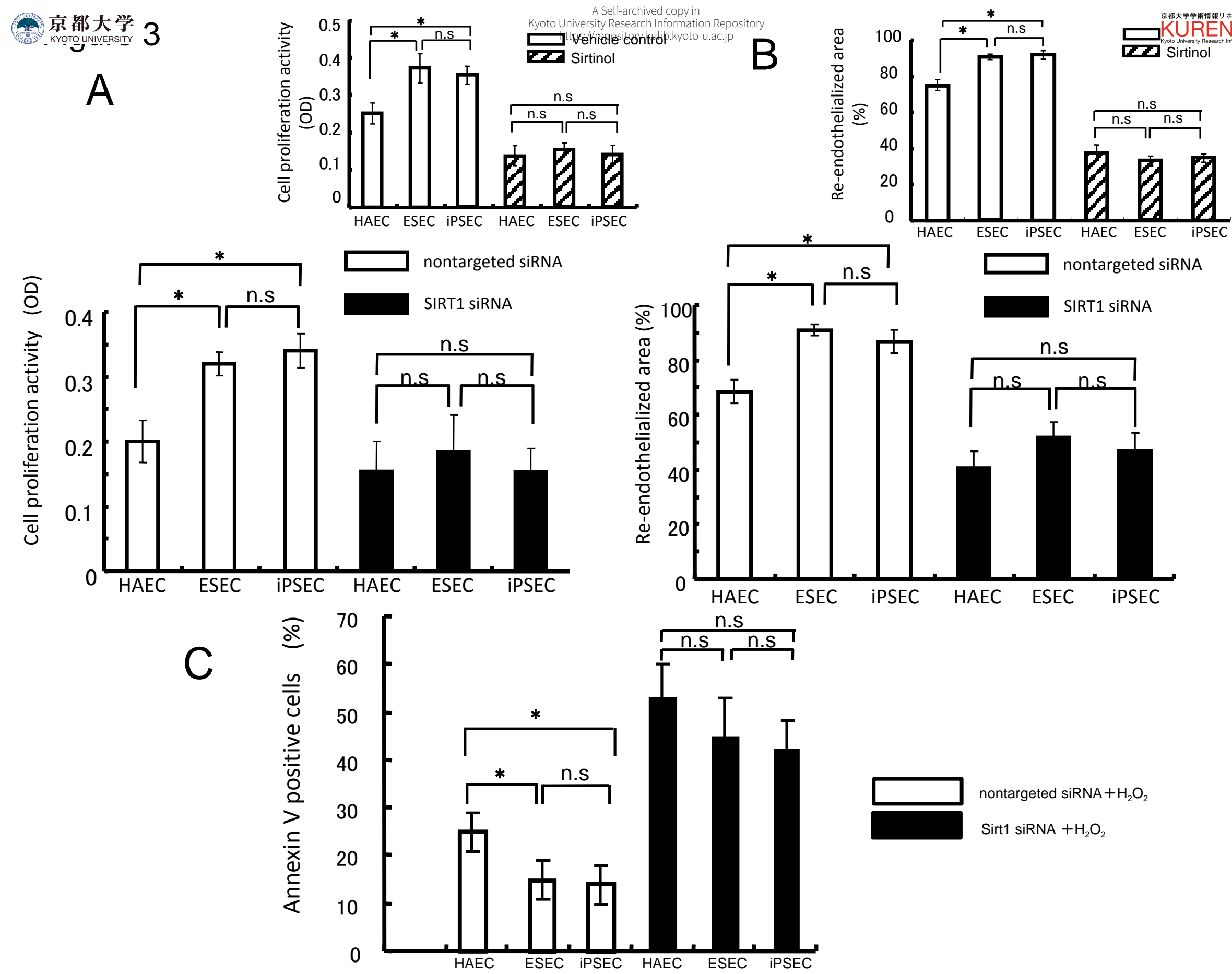
nontargeted SiRNA
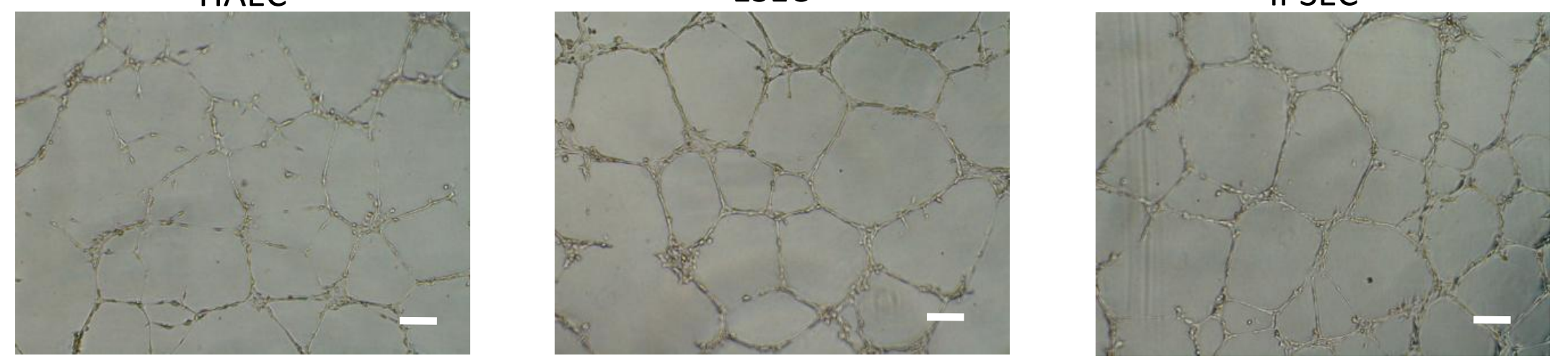

SIRT1

SiRNA
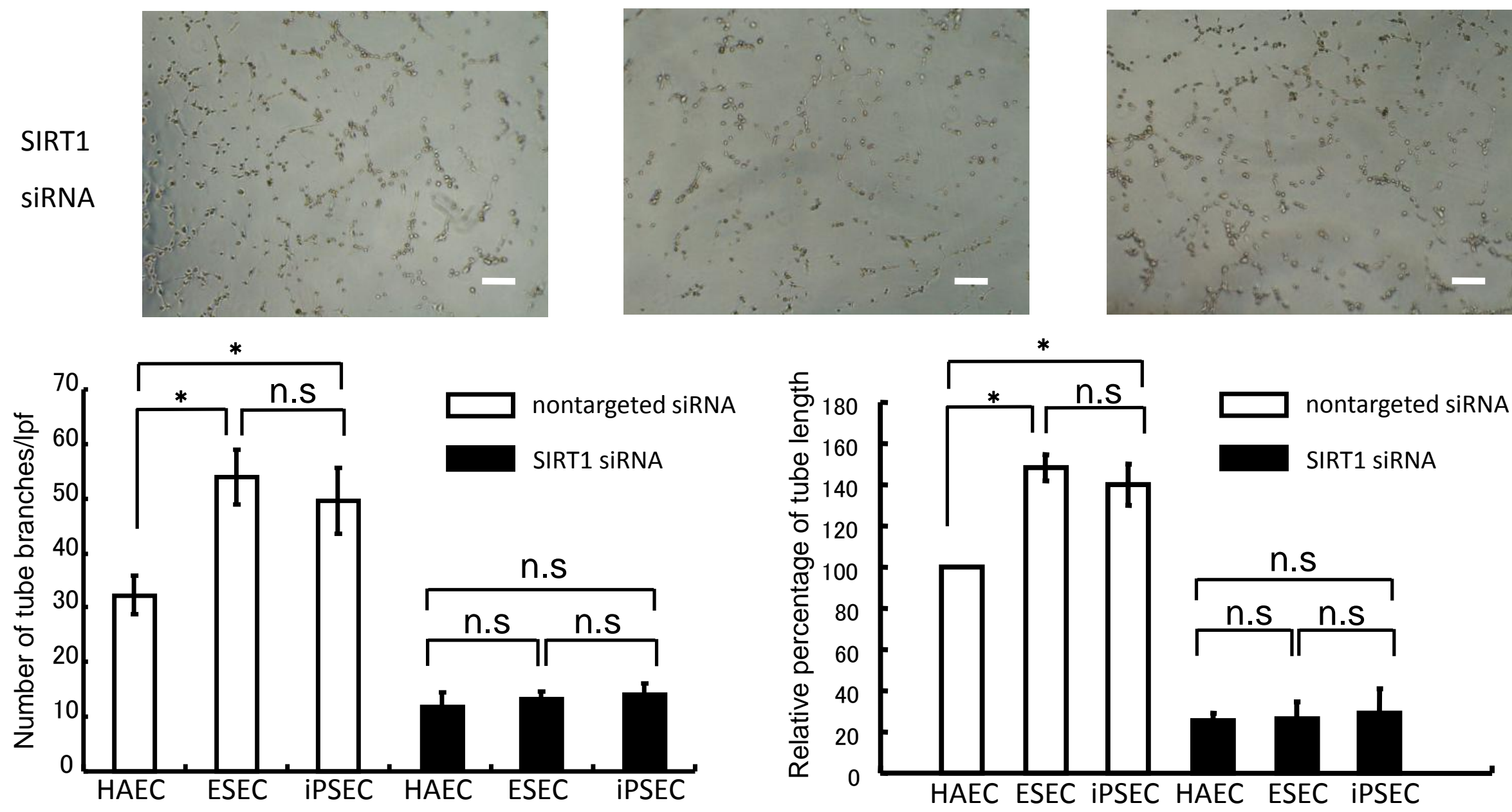